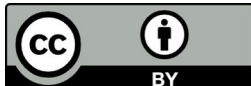

\title{
Las relaciones interpersonales y la calidad educativa
}

\author{
Interpersonal relationships and educational quality
}

Relações interpessoais e qualidade educacional

\section{ARTÍCULO GENERAL}

\author{
Jesús Nery Ramírez Agurto \\ jheynery1983@,hotmail.com \\ https:/orcid.org/0000-0002-9014-4811 \\ Universidad César Vallejo - Chiclayo \\ Lambayeque - Perú
}

\author{
Jorge Tesén Arroyo \\ jorgetesen@,hotmail.com \\ https://orcid.org/0000-0003-4111-6846 \\ Universidad César Vallejo - Chiclayo \\ Lambayeque - Perú
}

Recibido 07 de Junio 2021 | Arbitrado y aceptado 25 de Agosto 2021 | Publicado en 04 Diciembre 2021

\section{RESUMEN}

El propósito del presente artículo es realizar una revisión sistemática de la literatura científica sobre las relaciones interpersonales para lograr la calidad educativa. La metodología empleada revisión sistemática nivel descriptivo y analítico, se usó la base de datos (Dialnet, Scielo, Redalyc, Latindex) y en el buscador Google Académico; utilizando palabras clave en español: relaciones humanas y calidad educativa. Asimismo, se han tomado artículos científicos originales y una tesis de los últimos cinco años (2016-2021) desde su publicación. Se concluye que la calidad educativa tiene relación directa y significativa con las relaciones interpersonales, que es un factor fundamental para la mejora de los aprendizajes, porque en las escuelas se generan espacios de interacciones y también porque la calidad educativa es un proceso constante y permanente que se requiere fortalecer las relaciones y para brindar un servicio educativo de calidad está limitado también por las relaciones interpersonales de los maestros porque son ellos también, uno de los beneficiarios y que al fortalecerse estas interacciones mejora su dimensión personal y su desempeño docente. Las habilidades sociales como: la empatía, solidaridad y la comunicación ayudada de la gestión del talento humano y la inteligencia emocional que se propicien e incluyan en los documentos de gestión escolar contribuyen a fortalecer las relaciones humanas por ende mejorar la calidad educativa

Palabras claves: relaciones humanas y calidad educativa.

\section{ABSTRACT}

The purpose of this article is to carry out a systematic review of the scientific literature on interpersonal relationships to achieve educational quality. The methodology used was a systematic review, descriptive and analytical level, the database (Dialnet, Scielo, Redalyc, Latindex) and the Google Scholar search engine were used; using keywords in Spanish: human relations and educational quality. Likewise, original scientific articles and a thesis have been taken from the last five years (2016-2021) since its publication. It is concluded that educational quality has a direct and significant relationship with interpersonal relationships, which is a fundamental factor for the improvement of learning, because interaction spaces are generated in schools and also because educational quality is a constant and permanent process that It is necessary to strengthen relationships and to provide a quality educational service is also limited by the interpersonal relationships of teachers because they are also one of the beneficiaries and that by strengthening these interactions, their personal dimension and their teaching performance improves. Social skills such as: empathy, solidarity and communication aided by the management of human talent and emotional intelligence that are promoted and included in school management documents contribute to strengthening human relations and therefore improve educational quality.

Keywords: human relations and educational quality.

\section{RESUMO}

O objetivo deste artigo é realizar uma revisão sistemática da literatura científica sobre relacionamentos interpessoais para alcançar a qualidade educacional. A metodologia utilizada foi uma revisão sistemática, em nível descritivo e analítico, utilizou-se a base de dados (Dialnet, Scielo, Redalyc, Latindex) e o buscador Google Acadêmico; usando palavras-chave em espanhol: relações humanas e qualidade educacional. Da mesma forma, artigos científicos originais e uma tese foram retirados dos últimos cinco anos (20162021) desde a sua publicação. Conclui-se que a qualidade educacional tem relação direta e significativa com as relações interpessoais, o que é fator fundamental para a melhoria da aprendizagem, pois são gerados espaços de interação nas escolas e também porque a qualidade educacional é um processo constante e permanente que é necessário fortalecer relacionamentos e prestar um serviço educacional de qualidade também é limitado pelas relações interpessoais dos professores, pois eles também são um dos beneficiários e que, fortalecendo essas interações, melhora sua dimensão pessoal e seu desempenho docente. Habilidades sociais como: empatia, solidariedade e comunicação auxiliadas pela gestão do talento humano e inteligência emocional que são promovidas e incluídas nos documentos de gestão escolar contribuem para fortalecer as relações humanas e, consequentemente, melhorar a qualidade educacional.

Palavras-chave: relações humanas e qualidade educacional. 


\section{Introducción}

Las escuelas contribuyen en la transformación de la sociedad, es por ello que existen países con más desarrollo, donde se prioriza el campo educativo, pero también observamos instituciones educativas con bajos niveles de logro, diversos aspectos influyen en dichos resultados, y uno de ello es la existencia de rupturas de relaciones interpersonales entre los agentes educativos que afecta a todos. Por eso, es necesario fortalecer las relaciones interpersonales porque son esenciales para un buen funcionamiento de las instituciones que repercuten en la calidad educativa.

Según los informes anuales de progreso en la consecución de las metas de la Agenda 2030 a nivel mundial, a finales del 2019, millones de niños y jóvenes seguían sin escolarizar y más de la mitad de los que asistían a la escuela no cumplían los criterios mínimos de las competencias de lectura y cálculo. El cierre de las escuelas por la presencia de la COVID-19 está deteriorando la calidad educativa, el desarrollo social y conductual de niños y jóvenes, está afectando a más del $90 \%$ de la población estudiantil mundial 1.500 millones de niños y jóvenes (United Nations, 2020).Estos datos muestran un problema educativo que ya se viene acarreando y que con la presencia de la pandemia ahonda aún más la situación, que será muy difícil de superar esta realidad.

En el presente año, según los informes anuales de progreso en la consecución de las metas de la Agenda 2030 a nivel mundial. Se estima que 101 millones más de niños y jóvenes que cursaban los grados primero a octavo quedaron por debajo del nivel mínimo de la competencia en lectura en 2020 debido a los efectos de la pandemia y se perdió los avances educativos logrados durante los últimos 20 años. Los niveles de la competencia en lectura podrían recuperarse en 2024, pero solo si se dedican esfuerzos excepcionales a la tarea mediante estrategias de repaso y recuperación (United Nations, 2021). Entonces, podemos afirmar que en el campo educativo se ha dado un retroceso grande y que existe mucho que obrar para poder recuperarse y lograr tener mejores resultados en la calidad educativa en el mundo.

Los niños de la primera infancia también son afectados, porque no pueden recibir educación preescolar a causa de la pandemia. Esto puede conducir a resultados irreversibles en sus aprendizajes y afectar para el resto de sus vidas, situación diferente pasaba en años anteriores, según los datos recogidos entre 2012-2020 en 76 países y territorios, en su mayoría de ingreso bajo y mediano, indican que sin diferencias significativas entre los sexos, 7 de cada 10 niños de 3 y 4 años estaban bien encaminados 
en su desarrollo (United Nations, 2021).La calidad de los aprendizajes de los preescolares limitará su futuro desarrollo personal.

Siguiendo con la misma temática, los países de América Latina y el Caribe, ya experimentaba una crisis de aprendizaje antes que la COVID-19 impactara a la región a los inicios del 2020, con severas consecuencias en los resultados educativos y de capital humano. Al evaluar los casi doce meses del cierre masivo de escuelas en toda la región, queda claro que esta situación puede poner en peligro los avances del desarrollo humano, generando impactos sobre los futuros niveles de productividad limitando el desarrollo de los países de nuestra América Latina (GRUPO BANCO MUNDIAL, 2021).

La calidad de la Educación Básica Regular en el Perú se evidencia según la evaluación censal publicada por el Ministerio de Educación el 02 de junio de 2019. Los datos corresponden a la evaluación muestral de 125,540 estudiantes de cuarto grado de nivel primaria en 4,799 instituciones educativas. En la zona rural en el área de matemática logró un $15.5 \%$ en el nivel satisfactorio, hubo una mejora de $2.5 \%$ puntos respecto de los resultados censales del año 2018. Mientras que en lectura, el nivel satisfactorio se obtuvo $11.4 \%$, lo que significa un retroceso de 1,6 puntos con relación al año 2018. Este diagnóstico revela la crisis educativa que tenemos como país y la pandemia provocó socavar más este problema (DEFESORIA DEL PUEBLO, 2020)

Padilla y Rodríguez (2019) en su investigación dan a conocer el comportamiento del clima de convivencia escolar y sus dimensiones, constituido en uno de los otros indicadores de calidad (OIC) trascendentales para dar cuenta de las condiciones en que se suscita el aprendizaje. Concluyen que, los otros indicadores de calidad realza la necesidad de fortalecer el educar para vivir en comunidad, principalmente cuando las percepciones de los actores están fuertemente gobernadas por climas de convivencia deteriorada. Este clima de convivencia es la que genera que el aprendizaje se vea rezagado.

Fernández (2020) describe las características propias del clima de relaciones interpersonales existentes en el aula para el aprendizaje de estudiantes de los Colegios de la Ciudad de Pilar de Paraguay. El diseño empleado fue descriptivo,en ese mismo sentido, respecto a la variable relaciones interpersonales, la motivación que genera al tener favorables relaciones en el siguiente orden: $62 \%$ siempre, $19 \%$ a veces y el $19 \%$ nunca. Así también, existe un porcentaje elevado del buen desempeño académico que consisite en el $69 \%$. En el contexto educativo son necesarios ambientes adecuados de interacción 
que favorezca el aprendizaje efectivo en los alumnos gracias a las relaciones armoniosas que se generen.

Palacios, Damián \& Damián (2020) realizaron una investigación, que tuvo por objetivo establecer la relación entre el clima organizacional y la calidad de servicio de la institución educativa № 101 "Shuji Kitamura" del distrito de Santa Anita, Perú en el año 2017. Como instrumento, se utilizó el cuestionario de escala de Likert. El método es descriptivo y el diseño no experimental. Llega a concluir que, el clima organizacional influye significativamente en la calidad del servicio educativo con un coeficiente de significancia bilateral para la prueba Chi-cuadrado de 0.013 , indicando una fuerte asociación, además existe relación directa y significativa entre el clima organizacional y la calidad de servicio de la Institución.

Heredia et al. (2020) determina el nivel de satisfacción de los estudiantes como indicador de calidad en una institución educativa en Piura. El estudio es descriptivo, su población estuvo constituida por los estudiantes de una institución educativa privada de la región Piura, siendo un total de 465, como instrumento se usó el cuestionario. Arriban a la conclusión que el nivel de satisfacción de los estudiantes de la institución educativa acerca del servicio de los docentes, dio un resultado positivo, las metodologías de enseñanza utilizadas para los estudiantes son muy valoradas que contribuyen a su formación escolar y a su desarrollo como persona.

La teoría de las relaciones humanas tiene sus orígenes en la necesidad de humanizar y democratizar la admistración, además se desarrolló las ciencias huamanas, especificamente la Psicología y sus primeras aplicaciones en las organizaciones industriales, demostró lo indadecuado de los principos de la Teoría Clasica, gracias a Elton Mayo, Dewey y Lewin, la Sociología de Pareto y Hawthorne. La Teoria de las Relaciones Humanas ve a una organización como un grupo de personas que propicia confianza, considera la dinámica grupal e interpersonal, delega autoridad, prioriza a la personas y las relaciones que se generan entre ellas (Chiaverato, 2006).

Las relaciones humanas tienen como propósitos fundamentales: el desarrollo del ser humano, la productividad en el trabajo y la satisfacción personal dentro de la organización y la sociedad. Además, la búsqueda de soluciones a las múltiples frustraciones de la vida, así como la a identificación de las necesidades individuales y colectivas de las personas, dándoles posibles alternativas, como también fomentar la participación, integración y comunicación entre los seres humanos y finalmente alcanzar 
los objetivos de la empresa y satisfacer las necesidades humanas (Genao, Pérez, \& Castro, 2014).

Las habilidades sociales son una las conductas observables, pero también de pensamientos y emociones que nos permiten mantener relaciones interpersonales de forma satisfactoria, que consigamos un máximo de beneficios y son importantes porque nos genera bienestar, incrementa nuestra calidad de vida en la medida que nos ayuda a sentirnos bien y a obtener lo que queremos (Roca, 2014).

Las relaciones interpersonales es complejo de definir porque tenemos diversas concepciones, por lo que sí queda claro, que las relaciones interpersonales son interacciónes recíproca entre dos o más personas y estas interacciones suceden a largo de nuestra vida construyendo nuestra personalidad. Prieto (2016) define a las relaciones humanas como "el arte de conseguir lo que uno quiere sin lesionar los derechos de los demás"(p.63). Entendido como una habilidad compleja de lograr los objetivos, para ello usamos la comunicación efectiva, la empatía, el respeto, la tolerancia y la influencia en los demás.

El clima organizacional es una dimensión de calidad de vida laboral y tiene gran incidencia en la la productividad y el desarrollo del talento huamano de una entidad. Donde se identifique aquellas áreas de conflicto y herramientas primordiales para implementar la intervención necesaria de forma que se realice un cambio planificado que produzca un mayor satisfacción y productividad. Desde el punto de vista de la psicoligía laboral, es la influencia del ambiente del trabajo sobre la conducta de los miembros (López, 2001).

Con relación a la calidad educativa, Aguerrondo plantea el nuevo paradigma "Educación para Todos". La calidad en educación se puede definir por lo que se llaman los 7 lenguajes de la modernidad al terminar su educación obligatoria hayan logrado: altas competencias en lectura y escritura, cálculo matemático y resolución de problemas, expresión escrita, capacidad para analizar el entorno social y comportarse éticamente, capacidad para la recepción crítica de los medios de comunicación social, planear, trabajar y decidir en grupo, finalmente la capacidad para ubicar, acceder y usar mejor la información acumulada. Esto modifica lo que hay que aprender y enseñar para ser una sociedad competitiva y ser empleables (Aguerrondo, 1999). 
La Ley General de Educación (2003) define la calidad educativa como "el nivel óptimo de formación que deben alcanzar las personas para enfrentar los retos del desarrollo humano, ejercer su cuidadanía y continuar aprendiendo toda la vida (p.4).Este nivel óptimo es el valor que nos hace mejores seres humanos, ciudadanos competentes en todas sus dimensiones como personas logrando así el progreso y prosperidad para ellos y para el país.

La calidad es un proceso constante y permanente, ve a una organización como un proceso que está constituida fundamentalmente por relaciones. De estas relaciones, dos son las más importantes: las relaciones con los beneficiarios (alumnos y padres, fundamentalmente) y las relaciones entre quienes en ella trabajan. Mejorar la calidad significa trabajar en relaciones y si algo sale mal en un área, repercute en toda la organización (Schemelkes, 2010).

El objetivo del presente estudio es realizar una revisión sistemática de la literatura científica sobre las relaciones interpersonales para la calidad educativa y como objetivos específicos: identificar artículos científicos originales que tienen que ver las relaciones interpersonales y la influencia en la calidad educativa, analizar los aspectos relevantes de las investigaciones científicas y señalar los factores que influyen en las relaciones interpersonales para la mejora de calidad educativa.

El presente trabajo se justifica en el campo educativo y principalmente en las instituciones educativas, el capital humano son los que sinercian el aprendizaje y en las interaciones saludables se fortalecen las buenas relacionanes humanas, situación contraria pasa cuando se debilitan generando un ambiente de poca productividad o un mal servicio educativo, repercutiendo principalmente en la calidad de los aprendizajes de los alumnos; convivencia en ambientes hostiles, insatisfacción, pérdida de los talentos humanos y bajos logros institucionales. Entonces surge la necesidad de fortalecer, propiciar y mantener los vínculos humanos de las instituciones educativas porque nos agentes de motivación para su funcionamiento exitoso de las intituciones educativas.

\section{Materiales y métodos}

La búsqueda de información se realizó en los meses de junio y julio de 2021 en distintas bases de datos (Dialnet, Scielo, Redalyc, Latindex) y en el buscador Google Académico; utilizando palabras clave en español: relaciones humanas y calidad educativa,. Asimismo, se han tomado artículos científicos originales de las revistas 
científicas de los últimos cinco años (2016 - 2021) desde su publicación, el proceso de la filtración se descartó a los artículos que están relacionados con el tema las relaciones interpersonales, la calidad educativa y la confiabilidad de los resultados.

En el procesamiento y sistematización de la información se utilizó el método descriptivo y analítico, cual permitió la revisión sistémica de 14 fuentes seleccionadas y se organizó una tabla de análisis y síntesis donde se incluyeron campos temáticos como: Título del documento, autor y año, tipo de artículo, revista y Link o DOI.

\section{Resultados}

Los hallazgos encontrados en el análisis de 13 artículos científicos y 1 tesis relacionada con la temática, se obtuvieron los siguientes subtemas: habilidades sociales, clima organizacional, educación en valores, calidad educativa, relaciones personales y calidad del servicio educativo.

\section{Tabla 1}

Análisis y síntesis de los artículos científicos estudiados; considerando títulos, autores, años, revistas; entre otros.

\begin{tabular}{|c|c|c|c|c|c|}
\hline $\mathrm{N}^{\circ}$ & Título de documento & Autor / Año & $\begin{array}{l}\text { Tipo de } \\
\text { artículo }\end{array}$ & Revista & Link o DOI \\
\hline 1 & $\begin{array}{l}\text { Habilidades sociales } \\
\text { relevantes: percepciones de } \\
\text { múltiples actores educativos }\end{array}$ & $\begin{array}{l}\text { Tapia \& } \\
\text { Cubo, } 2017\end{array}$ & $\begin{array}{l}\text { Artículo de } \\
\text { investigación }\end{array}$ & $\begin{array}{l}\text { MAGIS. Revista } \\
\text { Internacional de } \\
\text { Investigación en } \\
\text { Educación }\end{array}$ & $\begin{array}{l}\frac{\text { https://www.re }}{\text { dalyc.org/pdf/2 }} \\
\underline{\text { 810/28105267 }} \\
\underline{\text { 8007.pdf }}\end{array}$ \\
\hline 2 & $\begin{array}{l}\text { Más confianza para una mejor } \\
\text { escuela: el valor de las } \\
\text { relaciones interpersonales entre } \\
\text { profesores y director }\end{array}$ & $\begin{array}{l}\text { Razeto, } \\
2017\end{array}$ & $\begin{array}{l}\text { Artículo de } \\
\text { investigación }\end{array}$ & $\begin{array}{l}\text { Cuadernos de la } \\
\text { investigación } \\
\text { Educativa. }\end{array}$ & $\begin{array}{l}\text { http://dx.doi.or } \\
\text { g/10.18861/cie } \\
\text { d.2017.8.1.263 } \\
9\end{array}$ \\
\hline 3 & $\begin{array}{l}\text { Relaciones interpersonales y } \\
\text { situaciones de convivencia en el } \\
\text { aula universitaria }\end{array}$ & $\begin{array}{l}\text { Loáiciga, } \\
2020\end{array}$ & $\begin{array}{l}\text { Artículo de } \\
\text { investigación }\end{array}$ & $\begin{array}{l}\text { Revista Académica } \\
\text { Arjé }\end{array}$ & $\begin{array}{l}\frac{\text { https://revistas. }}{\text { utn.ac.cr/index. }} \\
\underline{\text { php/arje/article }} \\
\underline{\text { view/240/216 }}\end{array}$ \\
\hline 4 & $\begin{array}{l}\text { Clima organizacional en los } \\
\text { colectivos académicos. }\end{array}$ & $\begin{array}{l}\text { Triana, et. } \\
\text { al., } 2019\end{array}$ & $\begin{array}{l}\text { Artículo de } \\
\text { investigación }\end{array}$ & $\begin{array}{l}\text { Revista Electrónica } \\
\text { Formación y Calidad } \\
\text { Educativa } \\
\text { (REFCalE) }\end{array}$ & $\begin{array}{l}\text { http://orcid.org } \\
\text { /0000-0001- } \\
\text { 8111-8307. }\end{array}$ \\
\hline
\end{tabular}


Clima institucional y desempeño de docentes en instituciones educativas de la Asociación

Educativa Adventista de Puno

6

Inteligencia emocional en educación superior: contribuciones a la calidad educativa.

$7 \quad$ Educación en valores para las relaciones interpersonales en estudiantes del colegio "San Isidro", Otuzco - 2019

Procesos de gestión del talento humano y relaciones

uancco interpersonales en la Unidad De Gestión Educativa Local de Yunguyo.

Calidad educativa y gestión institucional en la Universidad Nacional Intercultural de la Amazonía, Perú.

10 Liderazgo transformacional en las relaciones interpersonales y trabajo colaborativo de los directivos de la red 03, UGEL 04 Comas, 2020.

11 Liderazgo transformacional para mejorar la calidad educativa en el centro Santa María del proyecto Esperanza.

12 Relaciones interpersonales en el clima laboral de la universidad tecnológica del Chocó Diego

Luis Córdoba.

13 Influencia de las relaciones interpersonales en la calidad del servicio educativo.

14 Influencia del clima institucional en la calidad del servicio educativo de la Institución Educativa Ramón Castilla $\mathrm{N}^{\circ}$ 063, Cajamarca.

Enciso \&

Mamani,

2020 al., 2020

\&Vilca,

2020 al., 2020 2020 $\begin{aligned} \text { Ruíz, } 2019 & \begin{array}{l}\text { Artículo de } \\ \text { investigación }\end{array}\end{aligned}$

Lavado, et. Artículo de investigación

Revista TZHOECOEN

Artículo de investigación

Quispe, et.

Artículo de investigación

Revista científica, https://doi.org/ INICC-PERÚ

Revista Innova

http://revista.u nia.edu.pe/inde x.php/EDUCA CION

10.36996/delec tus.v3i2.48

Quiñones \& Artículo de Menacho, investigación

Batista, et. Artículo de Al., 2021 investigación

Revista

https://doi.org/ internacional. CIID $\quad 10.46785 /$ ciidj. Journal. v1i1.78

Revista UCE Ciencia http://ucecienci a.edu.do/index. php/OJS/articl e/view/229

Revista CES Derecho http://dx.doi.or $\mathrm{g} / 10.21615 /$ ces der.9.1.2.

al., 2018 investigación Artículo de
investigación

Flores, 2019 Tesis de

Repositorio

http://repositor investigación Institucional - UNFV io.unfv.edu.pe/ handle/UNFV/ $\underline{3529}$

Briones, Artículo de Revista. 2017 investigación PERSPECTIVA http://mail.upa gu.edu.pe/ojs/i ndex.php/PE/ar ticle/view/517 
Los 14 artículos están categorizados de la siguiente forma: calidad educativa y las relaciones interpersonales tenemos a Quispe et al. (2020), Quiñones et al. (2020) y Batista et al. (2020) en sus estudios reconocen que el liderazgo transformacional incide significativamente en las relaciones interpersonales y es un factor fundamental.

El clima organizacional y la calidad del servicio educativo Moreno, et al. (2018), Flores (2019) y Briones (2017) abordan la relación que existe entre el clima organizacional y la calidad del servicio educativo.

Un aspecto importante para las relaciones interpersonales son las habilidades sociales; a decir a (Tapia, 2017) y (Razeto, 2017). Según Huancco et al. (2020) las relaciones interpersonales influyen en el rendimiento del trabajador, sino ponen en peligro el mejoramiento de la calidad del servicio y el logro de los objetivos institucionales y finalmente los valores tienen influencia significativa en el fortalecimiento de relaciones (Ruiz, 2019) y (Lavado et al., 2020).

Como factores que truncan las buenas relaciones humanas; tenemos la falta o mala comunicación inteligencia emocional y la desconfianza limita las relaciones interpersonales Loáiciga (2020).

Finalmente, el clima institucional que existe en las instituciones educativas está vinculado estrechamente con el desempeño docente (Triana et al., 2019) y (Enciso et al., 2020).

\section{Discusión de los resultados}

La calidad educativa tiene relación directa y significativa con las relaciones interpersonales (Quispe et al., 2020). Para Quiñones et al. (2020) y Batista et al. (2020) reconocen que el liderazgo trasformacional contribuye a las relaciones interpersonales y es un factor fundamental para la mejora de los aprendizajes. Estos resultados se pueden corroborar con la Teoría de las Relaciones Humanas Chaverato (2006) quien considera a 
una organización como un grupo que prioriza a las personas y las relaciones que se generan entre ellas. En ese sentido, Schelmelkes (2010) sostiene que la calidad es un proceso constante y permanente que se requiere fortalecer las relaciones para mejorar la calidad de educación, no solo basta con esto, por eso, Aguerrondo (1999) plantea que para lograr la calidad educativa es necesario lograr las competencias, que hoy exige el mundo desde lograr las competencias de lectura hasta comportarse éticamente. En ese sentido, bajo lo mencionado anteriormente, confirmamos que las instituciones educativas son organizaciones que están en constante mejora siempre buscando la calidad y las relaciones interpersonales que son un elemento clave para mejorar la calidad educativa.

Moreno et al. (2018) y Flores (2019) reconocen que las relaciones interpersonales de los docentes influyen en la calidad del servicio educativo en la escuela. Esto lo podemos reafirmar con Schelmelkes (2010) que considera a la calidad como un proceso constante y permanente, y la organización que está constituida fundamentalmente por relaciones con los beneficiarios y entre quienes en ella trabajan. Entonces, si queremos calidad en las instituciones educativas está condicionada por las buenas o malas relaciones que se dan dentro de la comunidad y todo lo que sucede repercute en la institución educativa.

El clima organizacional tiene tendencia a ser desfavorable en la dimensión de relaciones interpersonales por lo que debe ser intervenida con prioridad para mejorarlo Triana, et al (2019). Por otro lado Briones (2017) y Enciso et al. (2020) considera que el clima institucional que existe en las instituciones está vinculado muy estrechamente con el desempeño docente que tiene relación directa con la calidad del servicio educativo. Estos estudios se pueden sustentar por López (2001) donde el clima organizacional es una dimensión de calidad de vida laboral y se refleja en la productividad y el desarrollo del talento humano generando influencia en el trabajo sobre la conducta de los miembros que 
pertenecen a la organización. Podemos concluir que las instituciones que tienen buenos climas institucionales ejercen influencia de forma positiva en la calidad de trabajo que realiza cada persona que la integra y repercute en la calidad educativa tanto en sus procesos como resultados.

Las habilidades sociales contribuyen a la enseñanza y aprendizaje Tapia (2017), las habilidades sociales como: la empatía, solidaridad y la comunicación fueron consideradas las más relevantes porque favorecen en la enseñanza, aprendizaje y la sana convivencia en el aula. Este resultado se sustenta por Roca (2014) donde las habilidades sociales nos permiten mantener relaciones interpersonales de forma satisfactoria e incrementar nuestra calidad de vida porque permite lograr lo que queremos. En ese sentido, confirmamos que las habilidades sociales favorecen a la enseñanza, aprendizaje en un ambiente que propicia una convivencia armoniosa lleno de interacciones que posibilitan lograr nuestros retos.

Otro aspecto a tener en cuenta, es la gestión del talento humano Huancco et al. (2020) concluyen que, el rendimiento del trabajador, permite el mejoramiento de la calidad del servicio y el logro de los objetivos institucionales. Por otro lado, también la confianza es ventajosa para las organizaciones escolares, Razeto (2017) y finalmente los valores que tienen influencia significativa en el fortalecimiento de relaciones Ruiz (2019) y Lavado, et al. (2020). Estos estudios se respalda con los propósitos fundamentales de las relaciones humanas Genao (2014) que busca el desarrollo del ser humano, la productividad en el trabajo para alcanzar los objetivos y más satisfacción personal dentro de la organización. Entonces al propiciar la confianza, la práctica de valores, al determinar y potencializar las habilidades de los integrantes de la comunidad educativa permite fortalecer nuestras relaciones, trae consigo grandes beneficios personales e institucionales reflejándose en los logros de la institución. 
Los factores que truncan las relaciones, según Loáiciga (2020) es la falta de comunicación o bien la mala comunicación de los equipos dentro del aula, la inteligencia emocional y la desconfianza limitan las relaciones interpersonales. Son aspectos limitantes que deterioran las interacciones que imposibilitan que las instituciones educativas logren sus mejores resultados y que requiere retomarlas en nuestros planes que puedan ser incluidos en los documentos de gestión.

\section{Conclusiones}

La calidad educativa tiene relación directa y significativa con las relaciones interpersonales que es un factor fundamental para la mejora de los aprendizajes porque en las escuelas se generan espacios de interacciones y también porque la calidad educativa es un proceso constante y permanente que se requiere fortalecer las relaciones para mejorar los aprendizajes y mucho más en este mundo de hoy que exige personas competentes para ello se requiere desarrollar diversidad de competencias que van desde lectura hasta comportarse éticamente, es por eso que las instituciones educativas están en constante mejora siempre buscando la calidad

La calidad del servicio educativo en la escuela está limitado también por las relaciones interpersonales de los maestros porque son ellos también, uno de los beneficiarios y que al fortalecerse estas interacciones, es el maestro el que recibe mayor ganancia tanto en su dimensión personal como profesional entonces la calidad en las instituciones educativas está condicionada por las buenas o malas relaciones que se dan dentro de la comunidad.

El clima institucional que existe en las instituciones está vinculado muy estrechamente con el desempeño del docente que tiene relación directa con la calidad del servicio educativo, esto se refleja en la productividad y el desarrollo del talento humano 
generando influencias positivas entre los miembros de la comunidad producto de ello, mejores logros institucionales que recaen en todos los beneficiarios.

Los factores que influyen en las relaciones interpersonales para la mejora de calidad educativa son las habilidades sociales como: la empatía, solidaridad y la comunicación que nos permiten mantener relaciones interpersonales de forma satisfactoria e incrementar nuestra calidad de vida porque permite lograr lo que queremos y favorecen a la enseñanza, aprendizaje en un ambiente que propicia una convivencia armoniosa ayudado de la inteligencia emocional, gestión del talento humano, la confianza en las organizaciones escolares y valores, todo esto permite mejorar la productividad en el trabajo para alcanzar los objetivos y más satisfacción personal dentro de la organización determinando y potencializando las habilidades de los integrantes de la comunidad educativa trae consigo grandes beneficios personales e institucionales reflejándose en los logros de la institución.

Los factores que truncan las relaciones interpersonales son la inadecuada comunicación, la desconfianza son limitantes que hace que se deterioren las interacciones repercutiendo en los resultados institucionales, por eso, requieren ser consideradas en nuestros planes que puedan ser incluidos en los documentos de gestión.

\section{Referencias}

Aguerrondo, I. (1999). El Nuevo Paradigma de la Educación para el siglo XXI. http://beu.extension.unicen.edu.ar/xmlui/bitstream/handle/123456789/58/El\%2 0Nuevo\%20Paradigma\%20de\%201a\%20Educaci\%C3\%B3n\%20para\%20el\%2 0siglo $\% 20 X X I . p d f$ sequence $=1$

BANCO MUNDIAL. (13 de abril de 2021). BANCO MUNDIAL-Educación. https://www.bancomundial.org/es/topic/education/overview\#1 
Batista Figuereo, A. P., López Avendaño, J. E., \& Díaz Valdés, T. (2021). Liderazgo transformacional para mejorar la calidad educativa en el centro Santa María del proyecto Esperanza. Revista UCE Ciencia., 9(1). http://uceciencia.edu.do/index.php/OJS/article/view/225

Briones Álvarez, M. R. (2017). Influencia del clima institucional en la calidad del servicio educativo de la institución educativa Ramón Castilla N 063,Cajamarca 2017. Rev. Perspectiva, $18(1)$ http://mail.upagu.edu.pe/ojs/index.php/PE/article/view/517/463

Chiaverato, D. (2006). Introducción a la teoría general de la administración (Séptima edición ed.). Editorial México. https://esmirnasite.files.wordpress.com/2017/07/i-admon-chiavenato.pdf

DEFESORIA DEL PUEBLO. (2020). La educación frente a la memergencia sanitaria. https://cdn.www.gob.pe/uploads/document/file/1252037/Serie\%20Informes\%2 0Especiales\%20N\%C2\%BA\%20027-2020-

DP\%20La\%20educaci\%C3\%B3n\%20frente $\% 20 \mathrm{a} \% 201 \mathrm{a} \% 20$ emergencia $\% 20$ sa nitaria.pdf

Enciso Cahuapaza, J., \& Mamani Benito, O. (2020). Clima institucional y desempeño de docentes en instituciones educativas de la Asociación Educativa Adventista de Puno. ÑAWPARISUN - Revista de Investigación Científica, 2(2). http://unaj.edu.pe/revista/index.php/vpin/article/view/94

Fernández Guillén, G. (2020). El clima de relaciones interpersonales existente para el aprendizajeen el aual de estudiantes de la ciudad del Pilar. Ciencia latina revista multidisciplinar, $4(2)$. https://ciencialatina.org/index.php/cienciala/article/view/192 
Flores Carlos, M. P. (2019). Influencia de las relaciones interpersonales en la calidad del servicio educativo. San Juan de Lurigancho. http://repositorio.unfv.edu.pe/handle/UNFV/3529

Genao, M. d., Pérez, A., \& Castro, R. (2014). Relaciones Humanas. UNAPECUniversidad

APEC.https://www.academia.edu/29111629/Libro_Relaciones_Humanas

GRUPO BANCO MUNDIAL. (2021). Actuemos ya para proteger el capital humano de nuestros niños. Banco Internacional de Reconstrucción y Fomento/ Banco Mundial. https://www.bancomundial.org/es/news/pressrelease/2021/03/17/hacer-frente-a-la-crisis-educativa-en-america-latina-y-elcaribe

Heredia Llatas, F. D., Ramos Farroñan, E. V., Ordinola Mora, A. P., \& Chuquicusma Juárez, D. (2020). Satisfacción de los estudiantes como indicador de calidad en una institucón educativa en la ciudad de Piura. Revista Conrado, 16(76). http://scielo.sld.cu/pdf/rc/v16n76/1990-8644-rc-16-76-245.pdf

Huancco Zela, B., \& Vilca Mamani, L. (2020). Procesos de gestión del talento humano y relaciones interpersonales en la Unidad De Gestión Educativa Local de $\begin{array}{lll}\text { Yunguyo. } & \text { Innova }\end{array}$ http://revista.unia.edu.pe/index.php/EDUCACION

Lavado Ríos, A. N., Rodríguez Nomura, H. E., Sánchez Pereda, S. A., \& Rodríguez Kong, M. P. (2020). Educación en valores para las relaciones interpersonales en estudiantes del colegio "San Isidro", Otuzco-2019. Revista Tzhoecoen, 12(1).http://revistas.uss.edu.pe/index.php/tzh/article/view/1256/1166

Ley general de educación .Nro. 28044. (2003). Obtenido de http://www.minedu.gob.pe/p/ley_general_de_educacion_28044.pdf 
Loáiciga Gutiérrez, J. (2020). Relaciones interpersonales y situaciones de convivencia en el aula universitaria. Revista Académica Arje, 3(1). Obtenido de https://revistas.utn.ac.cr/index.php/arje/article/view/240/216

López Paucar, G. (2001). Clima organizacional. Departamento Administrativo de la función pública. https://es.calameo.com/read/002047800d21749cbcaba

NACIONES UNIDAS. (2018). La Agenda 2030 y los Objetivos de Desarrollo Sostenible. Plublicaciones

CEPAL. https://repositorio.cepal.org/bitstream/handle/11362/40155/24/S1801141_es.pd $\mathrm{f}$

Padilla fuentes, G., \& Rodriguez Garcés, C. (2019). Clima de convivencia escolar en Chile: Un análisis desde el nuevo marco de medición de la calidad educativa. Revista Educación, 43(2). https://www.redalyc.org/articulo.oa?id=44058158045

Palacios Cruz, F. H., Damián Nuñez, E. F., \& Damián Nuñez, N. G. (2020). Relación entre el clima institucional y la calidad de servicio en una institución educativa del distrito Santa Anita, Perú, año2017. Revista Científica Tecnoógica UPSE, 7(1).https://incyt.upse.edu.ec/ciencia/revistas/index.php/rctu/article/view/502/4 51

Prieto Herrera, J. E. (2016). Gerencia Proactiva más allá de la visión empresarial (ECOE ediciones ed.). Obtenido de https://books.google.com.pe/books?id=laYwDgAAQBAJ\&pg=PT80\&dq=relac iones + humanas $\&$ hl $=$ es-419\&sa $=$ X\&ved $=2$ ahUKEwj9opY_ObxAhWT1JUCHbo0A5EQ6AF6BAgFEAI\#v=onepage\&q=relaciones $\% 20$ humanas\& $\mathrm{f}=$ false 
Quiñones Cuyubamba, S. M., \& Menacho Rivera, A. S. (2020). Liderazgo transformacional en las relaciones interpersonales y trabajo colaborativo de los directivos de la red 03, UGEL 04 Comas, 2020. CIIDJournal. REVISTA INTERNACIONAL MULTIDISCIPLINARIACIID, https://ciidjournal.com/index.php/abstract/article/view/78/73

Quispe Cutipa, W. A., Carola, Z. S., \& Sangama Sánchez, J. L. (2020). Calidad educativa y gestión institucional en la Universidad Nacional Intercultural de la Amazonía, Perú. Revista $\quad$ Delectus, $3(2) . \quad$ https://revista.iniccperu.edu.pe/index.php/delectus/article/view/48/78

Razeto, A. (2017). Más confianza para una mejor escuela: el valor de las relaciones interpersonales entre profesores y director. Cuadernos de Investigación Educativa, $\quad 8(1) . \quad \mathrm{https}: / /$ revistas.ort.edu.uy/cuadernos-de-investigacioneducativa/issue/view/233

Roca, E. (2014). Cómo mejorar tus habilidades sociales. Valencia: ACDE Ediciones. https://www.cop.es/colegiados/PV00520/pdf/Habilidades\%20socialesDale\%20una\%20mirada.pdf

Ruiz Segarra, M. I. (2019). Inteligencia emocinal en educación superior: contribuciones a la calidad educativa. Yanachana Revista Científica, 8(3). http://revistas.ulvr.edu.ec/index.php/yachana/article/view/608/351

Schemelkes, S. (2010). Hacia una mejor calidad de nuestras escuelas. México: Editorial Acude.

https://docs.google.com/file/d/1AtiPjIgCSp1UNRq6Wfpyim80tbG4K88Fwnm 4bUdYQp6h4mIRpe24E19rRJg1/edit?pref=2\&pli=1

Sorleidy Indira Moreno Perea, S. I., \& Elkin Olaguer Perez Sanchez, E. O. (2018). Relaciones interpersonales en el clima laboral de la universidad tecnológica del 
Chocó Diego Luis Córdoba. Revista CES Derecho, 9(1). http://www.scielo.org.co/pdf/cesd/v9n1/2145-7719-cesd-9-01-13.pdf

Tapia Gutiérrez, C. P., \& Cubo Delgado, S. (2017). Habilidades sociales relevantes: percepciones de multiples actores educativos. MAGIS.Revista Internacional de $\begin{array}{lll}\text { Investigación } & \text { en } & \text { Educación, }\end{array}$ https://www.redalyc.org/pdf/2810/281052678007.pdf

Triana Hernández, B. M., Alarcón Ortiz, R., \& Quevedo Cubillos, M. N. (2019). Clima organizacional en los colectivos académicos. Revista Electrònica Formaciòn y Calidad

Educativa, $7(2)$. https://www.researchgate.net/publication/335755669_EL_CLIMA_ORGANIZ ACIONAL_EN_LOS_COLECTIVOS_DE_ANOS_ACADEMICOS

United Nations. (2021). Progress towards the Sustainable Development Goals : report of the Secretary-General. https://digitallibrary.un.org/record/3930067? $\ln =$ en

United Nations. (2020). Progress towards the Sustainable Development Goals : report of the Secretary-General.https://digitallibrary.un.org/record/3865828? $1 \mathrm{n}=\mathrm{en}$ 